\title{
Structural and Aerodynamic Models in Nonlinear Flight Dynamics of Very Flexible Aircraft
}

\author{
Rafael Palacios,,- Joseba Murua, $\_$and Robert Cook \\ Imperial College, London, England SW7 2AZ, United Kingdom
}

DOI: $10.2514 / 1 . J 050513$

\begin{abstract}
An evaluation of computational models is carried out for flight dynamics simulations on low-speed aircraft with very-flexible high-aspect ratio wings. Structural dynamic models include displacement-based, strain-based, and intrinsic (first-order) geometrically-nonlinear composite beams, while thin-strip and vortex lattice methods are considered for the unsteady aerodynamics. It is first shown that all different beam finite element models (previously derived in the literature from different assumptions) can be consistently obtained from a single set of equations. This approach has been used to expand existing strain-based models to include shear effects. Comparisons are made in terms of numerical efficiency and simplicity of integration in flexible aircraft flight dynamics studies. On the structural modeling, it was found that intrinsic solutions can be several times faster than conventional ones for aircraft-type geometries. For the aerodynamic modeling, thin-strip models based on indicial airfoil response are found to perform well in situations dominated by small amplitude dynamics around large quasi-static wing deflections, while large-amplitude wing dynamics require three-dimensional descriptions (e.g. vortex lattice).
\end{abstract}

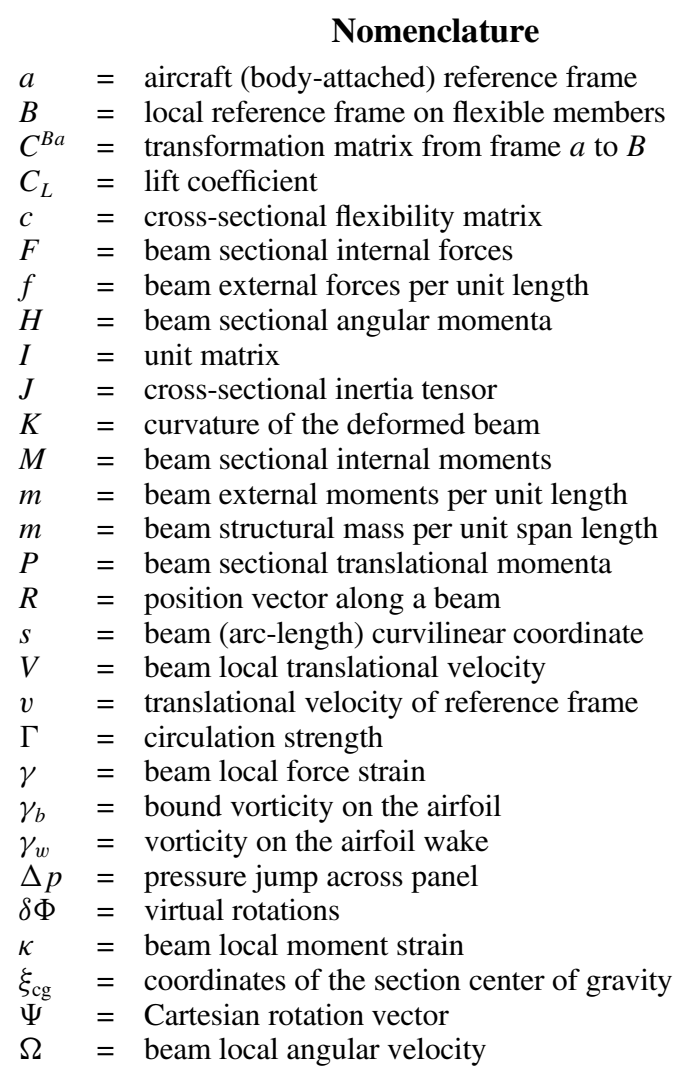

Presented in part as Paper 2009-2403 at the 50th AIAA/ASME/ASCE/ AHS/ASC Structures, Structural Dynamics, and Materials Conference, Palm Springs, CA, 4-7 May 2009; received 20 February 2010; revision received 6 August 2010; accepted for publication 9 August 2010 . Copyright $\odot 2010$ by Rafael Palacios, Joseba Murua, and Robert Cook. Published by the American Institute of Aeronautics and Astronautics, Inc., with permission. Copies of this paper may be made for personal or internal use, on condition that the copier pay the $\$ 10.00$ per-copy fee to the Copyright Clearance Center, Inc., 222 Rosewood Drive, Danvers, MA 01923; include the code 0001-1452/10 and $\$ 10.00$ in correspondence with the CCC.

*Lecturer, Department of Aeronautics, 355 Roderic Hill Building; rpalacio@imperial.ac.uk. Member AIAA.

${ }^{\dagger}$ Graduate Student, Department of Aeronautics, 352 Roderic Hill Building. Student Member AIAA.

${ }^{\ddagger}$ Graduate Student, Department of Aeronautics, 352 Roderic Hill Building. $\omega=$ angular velocity of reference frame

- $\quad=$ derivatives with respect to time, $t$

- $\quad=$ skew-symmetric (cross-product) operator

$\bullet^{\prime} \quad=$ derivatives with the curvilinear coordinate, $s$

\section{Introduction}

$\mathbf{T}$ HE studies in this paper are part of the development of an analysis framework for performance prediction and flightcontrol-system (FCS) design of ultra-long-endurance solar-powered unmanned aerial vehicles (UAVs). Examples of vehicles in this class are NASA's Helios, QinetiQ's Zephyr, and the proposed concepts for the Defense Advanced Research Projects Agency Vulture program. This is a new class of carbon-free airborne systems that may provide a cost-effective platform for a large number of tasks, from continuous earth and environmental observations to low-orbit data links. Solarpowered vehicles fill then the gap between satellites and conventional fuel-powered UAV, providing low-cost continuous coverage of a geographic area. As the power available from solar cells is very limited, these are airplanes with an extremely low weight and very high aspect ratio wings for maximum aerodynamic efficiency. Large lightweight UAVs have brought a new interest into the flight dynamics of flexible aircraft, including a need to expand quasi-static approaches for the evaluation of the basic aerodynamic derivatives of large aircraft [1]. In most cases the work has focused on the rigidbody equations augmented with linear structural models [2,3]. While this approach is valid in a majority of situations, it is not sufficient for a complete analysis of very flexible aircraft (VFA) with large changes of the inertia properties. In that case, nonlinear couplings arising from large wing deflections may play a critical role in the vehicle dynamics, as it was sadly illustrated by the mishap of NASA's Helios [4].

The major issues that need to be addressed in the development of an appropriate FCS design methodology for VFA include:

1) Analyses should accurately represent the large deformations of the very flexible primary structures.

2) They should consider aerodynamic characteristics determined by large motions of the wetted surfaces relative to mean flow speed.

3) The vehicle natural structural frequencies will be comparable to the time scales in the flight dynamics response, and thus the coupling between aeroelasticity and flight dynamics should be taken into account.

As all these problems are intrinsically nonlinear, performance prediction for long-endurance UAVs becomes a multidisciplinary 
problem involving the vehicle nonlinear structural, aerodynamics, and flight dynamics.

The pioneer work to include geometrically-nonlinear effects in the deformation of low-stiffness aircraft was conducted in the development of the Daedalus human-powered aircraft [5]. This aircraft had very restricted flight conditions and nonlinear effects were only included in the static aeroelastic analysis, while the dynamic response of the aircraft was based on linear models. Still, the large deformations had a large impact in the actual flight dynamics characteristics of the aircraft. Long-endurance aircraft will have to operate in a less restricted flight envelope than the Daedalus. For their design to be practical, aircraft need to be designed to withstand much higher levels of atmospheric turbulence, particularly for the mission segments in the lower atmosphere. For a VFA with high-aspect ratio wings, this often means dynamic loads that create rather large instantaneous wing deflections. Different groups [6-8] have developed models for such scenarios based on the coupling of geometricallynonlinear beams, unsteady thin-strip aerodynamics and the flight dynamics of the full vehicle. This allows capturing the basic physical phenomena with a relatively small size of the problem and has provided significant evidence on the large effect that structural deformations may have on the aircraft dynamics characteristics (e.g., on the short-period and phugoid modes). Wang et al. [9] later replaced the thin-strip model in [7] by three-dimensional aerodynamics based on the unsteady vortex lattice method (UVLM). A stripwise correction was introduced to account for airfoil stall and the model was applied to a flying-wing configuration. However, no comparisons were presented with coupled simulations based on twodimensional aerodynamics to quantify the improvement of the methodology.

Many research groups [7-11] have given substantial evidence of the most important aspects in which the response of VFA is affected by a nonlinear structural behavior, including the longitudinal stability characteristics, body-freedom flutter, response to gust and turbulence loads, and definition of control laws. A critical aspect for successful approaches lies in the identification of a reduced set of degrees of freedom able to account for the interactions between structural, aerodynamics and flight dynamics in long-term dynamic simulations (for instance, in analysis of complex maneuvers or optimal path tracking). This is particularly critical for future use of those models for robust flight-control system design for VFA. For the modeling of the structural dynamics, implicit [10] and strain-based [12] beam models are particularly suitable for this type of applications, dominated by large quasi-steady displacements: For static problems, they simply need the inversion of a constant matrix, and for dynamic problems with low-frequency content they will have very fast convergence rates. For them to be of practical use, they need to be accompanied by a high-fidelity dimensional reduction procedure $[13,14]$ from the actual three-dimensional solid mechanics problem. In the unsteady aerodynamics description, large wing excursions prevent the use of doublet-lattice and similar methods based on small displacements and flat wake assumptions. Alternative methods, such as the vortex lattice method (VLM) [15], have been then proposed. However, there needs to be a compromise among the number of aerodynamic states added to the solution and the accuracy improvements, as thin-strip methodologies [16-18] can be a more cost-effective solution than panel methods for unsteady computations on high-aspect ratio wings. Finally, the time integration of the coupled equations needs to be performed by an efficient yet accurate integration scheme for the resulting nonlinear system of equations [19].

This paper will investigate different alternatives of aerodynamics and structural dynamics models suitable for flight dynamics of VFA. First, different composite beam models will be developed from a common description of the geometrically-exact beam equations [20]. Three solution methods will be presented, based on finite element discretizations with different sets of independent degrees of freedom (displacements, strains, and internal force/velocities). The resulting models are generalizations of current approaches in the literature, and the common framework will be used to compare their performance and applicability. Analogously, unsteady thin-strip and vortex lattice methodologies will be briefly presented, and their application to slender wings with large displacements will be numerically investigated. However, details of the coupling into a full-aircraft aeroelastic model are not considered in this work and will appear in a separate paper.

\section{Structural Dynamic Modeling}

Composite beam models provide a very efficient way of capturing the dynamics of the different primary structures of high-aspect ratiowing vehicles. A typical representation, corresponding to a conventional wing-body-tail configuration, is shown in Fig. 1 .

Figure 1 also includes the different reference frames used in the analysis: $\bar{G}$ is an inertial coordinate system; $a$ is a (moving) bodyattached reference frame with translational and angular velocities $v_{a}$ and $\omega_{a}$, respectively, that will define the free-body dynamics of the vehicle; and $B$ is the local coordinate system along the deformed beam reference line. The structural model will be built from the equations of motion of a curved nonlinear beam of arc-length $l$ in the time interval $\left[t_{1}, t_{2}\right]$. They are obtained from Hamilton's principle applied on the homogenized composite structure and can be written in weak form, for prescribed motions of the global frame, $a$, as [20]

$$
\begin{aligned}
& \int_{t_{1}}^{t_{2}} \int_{0}^{l}\left[\delta V_{B}^{T} P_{B}+\delta \Omega_{B}^{T} H_{B}-\delta \gamma^{T} F_{B}-\delta \kappa^{T} M_{B}\right. \\
& \left.\quad+\delta R_{a}^{T} f_{a}+\delta \Phi_{B}^{T} m_{B}\right] \mathrm{d} s \mathrm{~d} t=0
\end{aligned}
$$

Subindexes are used to indicate the coordinate system in which each vector magnitude is projected. Linear constitutive relations are assumed at an arbitrary location of the beam reference line and for arbitrary distribution of the anisotropic (composite) material [13]:

$$
\begin{aligned}
& P_{B}=m V_{B}-m \tilde{\xi}_{\mathrm{cg}} \Omega_{B}, \quad \text { and } \quad \begin{array}{l}
\gamma=c_{\gamma f} F_{B}+c_{\gamma m} M_{B}, \\
H_{B}=m \tilde{\xi}_{\mathrm{cg}} V_{B}+J \Omega_{B},
\end{array} \quad c_{\kappa f} F_{B}+c_{\kappa m} M_{B}
\end{aligned}
$$

Equations (1) and (2) and are solved together with the nonlinear velocity-displacement and the strain-displacement kinematic relations [20]:

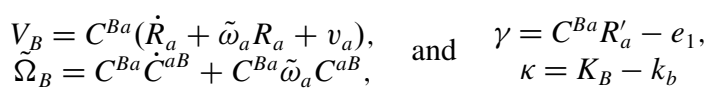

where $K_{B}=\operatorname{vect}\left(C^{B a} C^{a B^{\prime}}\right)$ is the current curvature, $k_{b}$ is the initial curvature (in its components in the local undeformed frame, $b$ ) and $e_{1}=\left[\begin{array}{lll}1 & 0 & 0\end{array}\right]^{T}$. Equations $(\underline{1}-\underline{3})$ are complemented by the corresponding initial and boundary conditions. If $v_{a}$ and $\omega_{a}$ are not prescribed, additional equations are needed to describe the free-body motions of the vehicle. They can be written as

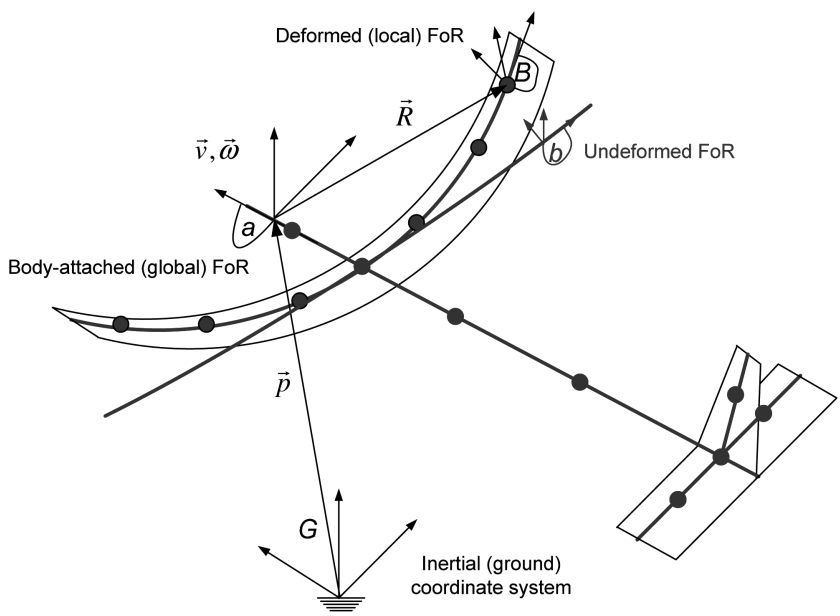

Fig. 1 Beam model of a wing-body-tail configuration, including the reference frames used in the analysis. 


$$
\begin{aligned}
& \left(\frac{\mathrm{d}}{\mathrm{d} t}+\omega_{a}\right) \int_{0}^{l} C^{a B} P_{B} \mathrm{~d} s=\int_{0}^{l} f_{a} \mathrm{~d} s \\
& \left(\frac{\mathrm{d}}{\mathrm{d} t}+\omega_{a}\right) \int_{0}^{l}\left(C^{a B} H_{B}+\tilde{R}_{a} C^{a B} P_{B}\right) \mathrm{d} s=\int_{0}^{l} m_{a} \mathrm{~d} s
\end{aligned}
$$

where the integrals on the left hand side are the total instantaneous translational and angular momenta of the deforming body in their components in the moving frame. Equations (1- 1 ), with spanwise forces, $f_{a}$, and moments, $m_{a}=C^{a B} m_{B}$, given by the corresponding aerodynamic model, provide a compact description of the flight dynamics of the flexible aircraft. Several solution procedures can be devised to solve the problem defined by Eqs. (1-4). This work will consider three alternative solutions, all based on the finite element method, but with each one using a different selection of independent degrees of freedom, namely, displacements, strains, and combined velocities and internal forces (the intrinsic solution).

\section{A. Formulation in Displacements and Rotations}

This is the solution process most commonly found in the literature, for which a excellent description (and an extensive literature survey can be found) in [21]. However, most references do not consider anisotropic material properties, as it is done here. First, Eq. (1) is written in strong form [20]:

$$
\begin{aligned}
& \left(\frac{\mathrm{d}}{\mathrm{d} t}+\tilde{\Omega}_{B}\right) P_{B}=\left(\frac{\mathrm{d}}{\mathrm{d} x}+\tilde{K}_{B}\right) F_{B}+f_{B} \\
& \left(\frac{\mathrm{d}}{\mathrm{d} t}+\tilde{\Omega}_{B}\right) H_{B}+\tilde{V}_{B} P_{B}=\left(\frac{\mathrm{d}}{\mathrm{d} x}+\tilde{K}_{B}\right) M_{B}+\left(\tilde{e}_{1}+\tilde{\gamma}\right) F_{B}+m_{B}
\end{aligned}
$$

A parameterization of the finite rotation is now needed (Euler or Bryant angles, Rodrigues parameters, quaternions, etc.). We have chosen, as in [21] and others, the Cartesian rotation vector, $\Psi$, in the rotation from the reference coordinate system $(a)$ to the local deformed frame $(B)$. The Cartesian rotation vector introduces the minimum number of states (three) without introducing singularities. Next, we substitute Eq. (3) into Eq. (2) and then into the equilibrium Eqs. (5) to define a closed set of equations on the state variable $\eta^{T}=\left[\begin{array}{ll}R_{a}^{T} & \Psi^{T}\end{array}\right]$, which is then approximated by a finite element discretization, i.e., $\eta=N \bar{\eta}$, where $\bar{\eta}$ is the set of discrete degrees of freedom and $N$ the corresponding shape functions. In this work twoand three-noded curved beam elements have been implemented. With these definitions, Eq. (5) can be written as

$$
\mathcal{M}_{\eta}(\bar{\eta}) \ddot{\bar{\eta}}+m_{a}(\bar{\eta})\left\{\begin{array}{c}
\dot{v}_{a} \\
\dot{\omega}_{a}
\end{array}\right\}+\mathcal{F}_{\text {gyr }}\left(\bar{\eta}, \dot{\bar{\eta}}, v_{a}, \omega_{a}\right)+\mathcal{F}_{\text {stif }}(\bar{\eta})=\mathcal{F}_{\text {ext }}
$$

where $\mathcal{M}_{\eta}$ is the discrete mass matrix and $\mathcal{F}_{\text {gyr }}, \mathcal{F}_{\text {stif }}$ and $\mathcal{F}_{\text {ext }}$ are the discrete gyroscopic, stiffness, and external generalized forces, respectively. This is the conventional approach to create a geometrically-nonlinear beam element (what will named the $d$-beam element), whose details have been extensively studied in the literature $[21,22]$. It has several advantages: it directly provides the solution in the problem physical degrees of freedom for aeroelastic or flexible-body dynamic problems, its linearized version yields the usual symmetric matrices, and a number of efficient solution algorithms (Newmark, HHT, Generalized- $\alpha$ ), are readily available. However, solving directly the rotational degree of freedom is a costly numerical exercise. The number of operations to build the functionals in Eq. (6) is relatively high and convergence of implicit algorithms based on Newton-Raphson procedures is relatively slow. In addition to this, the mass matrices and the different functionals need to be regularly updated in the solution.

\section{B. Strain-Based Beam Element}

An alternative solution path is obtained by taking the force and moment strains defined in Eq. (3) as the independent variables in the problem and define them to be piecewise constant in the discretization of the reference line. This procedure was first introduced in [12] from geometrical arguments and assuming zero shear strains, and it has been later used in a number of studies $[8,11,19,23]$. We will present an alternative procedure to derive the strain-based dynamic beam equations based on the analytical integration of the full strain-displacement relations. For that purpose, the second set of Eqs. (3) is first rewritten as

$$
R_{a}^{\prime}=C^{a B}\left(e_{1}+\gamma\right), \quad C^{a B^{\prime}}=C^{a B} \tilde{K}_{B}
$$

If $\gamma=\gamma_{n}$ and $\kappa=\kappa_{n}$ are constant within the segment $s_{n-1} \leq s<s_{n}$, as it was assumed in [12], Eq. (7) can be easily piecewise integrated using the properties of the exponential map as

$$
\begin{aligned}
& R_{a}(s)=R_{a}\left(s_{n-1}\right)+C^{a B}\left(s_{n-1}\right) \mathcal{H}_{s}^{1}\left(e_{1}+\gamma_{n}\right) \\
& C^{a B}(s)=C^{a B}\left(s_{n-1}\right) \mathcal{H}_{s}^{0}
\end{aligned}
$$

where $\mathcal{H}_{s}^{0}=\mathcal{H}^{0}\left(\kappa_{n}, s-s_{n-1}\right)$ and $\mathcal{H}_{s}^{1}=\mathcal{H}^{1}\left(\kappa_{n}, s-s_{n-1}\right)$, with the operators defined as

$$
\begin{aligned}
& \mathcal{H}^{0}(\kappa, \Delta s)=e^{\widetilde{\Delta \Psi}}=I+\frac{\sin (\Delta \phi)}{\Delta \phi} \widetilde{\Delta \Psi}+\frac{1-\cos (\Delta \phi)}{\Delta \phi^{2}} \widetilde{\Delta \Psi} \widetilde{\Delta \Psi} \\
& \mathcal{H}^{1}(\kappa, \Delta s)=\Delta s\left(I+\frac{1-\cos (\Delta \phi)}{\Delta \phi^{2}} \widetilde{\Delta \Psi}\right. \\
& \left.\quad+\frac{\Delta \phi-\sin (\Delta \phi)}{\Delta \phi^{3}} \widetilde{\Delta \Psi} \widetilde{\Delta \Psi}\right)
\end{aligned}
$$

with $\Delta \phi=\|\Delta \Psi\|$ and $\Delta \Psi=K_{B} \Delta s=\left(k_{b}+\kappa\right) \Delta s$. Note that there is no need to assume that shear strains are zero $\left(\gamma_{12}=0, \gamma_{13}=0\right)$ as it was done in [12], although that approximation can be often justified. Equations (8) therefore describes the kinematics of the reference line using constant-strain elements of length $\Delta s_{n}=s_{n}-s_{n-1}$ : For a given value of the position vector, $R_{a 0}$, and orientation, $C_{0}^{a B}$, at $s=s_{0}$ (i.e., the boundary values), Eqs. (8) define a recursive procedure to obtain the position vector and orientation within the $n$th element (i.e., $s_{n-1} \leq s<s_{n}$ ) as function of the current force and moment strains along the beam. Discontinuities in curvature of the discrete reference line can be easily included as additional (constant) rotations between the elements that are linked to a given node. For closed kinematic chains, these equations need to be complemented by the appropriate constraints, which can be defined by Lagrange multipliers [23], but this is not relevant in typical aircraft geometries and will not be discussed here. Without loss of generality, we can take as the reference frame to describe the beam kinematics its local frame at $s=0$, that is, $a \equiv B_{0}$, then it is $R_{a 0}=0$ and $C_{0}^{B a}=I$. Differentiation of Eqs. (8), gives the virtual displacements and rotations as function of the virtual strains, which in symbolic form can be written as

$$
\begin{aligned}
& \delta R_{a}(s)=\mathcal{H}_{\gamma}^{1}(\bar{\kappa}, s) \delta \bar{\gamma}+\mathcal{H}_{\kappa}^{1}(\bar{\gamma}, \bar{\kappa}, s) \delta \bar{\kappa} \\
& \delta C^{a B}=\mathcal{H}_{\kappa}^{0}(\bar{\kappa}, s) \delta \bar{\kappa}
\end{aligned}
$$

where we have introduced the following vectors of the independent degrees of freedom in the discretization of the problem $\bar{\gamma}^{T}=\left\{\gamma_{1}, \ldots, \quad \gamma_{N}\right\}, \bar{\kappa}^{T}=\left\{\kappa_{1}, \ldots, \quad \kappa_{N}\right\}$. Note that $\mathcal{H}_{\kappa}^{0}$ is a third-order tensor. Similar expressions are obtained for the time derivatives of the position vector and rotation matrix, as

$$
\begin{aligned}
& \dot{R}_{a}=\mathcal{H}_{\gamma}^{1} \dot{\bar{\gamma}}+\mathcal{H}_{\kappa}^{1} \dot{\bar{\kappa}}, \quad \ddot{R}_{a}=\mathcal{H}_{\gamma}^{1} \ddot{\bar{\gamma}}+\mathcal{H}_{\kappa}^{1} \ddot{\bar{\kappa}}+\dot{\mathcal{H}}_{\gamma}^{1} \dot{\bar{\gamma}}+\dot{\mathcal{H}}_{\kappa}^{1} \dot{\bar{\kappa}}, \\
& \dot{C}^{a B}=\mathcal{H}_{\kappa}^{0} \dot{\bar{\kappa}}, \quad \ddot{C}^{a B}=\mathcal{H}_{\kappa}^{0} \ddot{\overline{\bar{\kappa}}}+\dot{\mathcal{H}}_{\kappa}^{0} \dot{\bar{\kappa}}
\end{aligned}
$$

For simplicity, it is assumed now that the beam reference line is located at the center of mass of each cross section $\left(\xi_{\mathrm{cg}}=0\right)$. Under that assumption, Eq. (1) is written as

$$
\begin{gathered}
\int_{t_{1}}^{t_{2}} \int_{0}^{l}\left\{\delta R_{a}^{T}\left(m \dot{V}_{a}+m \tilde{\omega}_{a} V_{a}-f_{a}\right)+\delta \gamma^{T} F_{B}+\delta \kappa^{T} M_{B}\right. \\
\left.+-\frac{1}{2} \operatorname{tr}\left[\delta C^{B a} C^{a B}\left(J \tilde{\dot{\Omega}}_{B}+J \tilde{\Omega}_{B} \Omega_{B}-\tilde{m}_{B}\right)\right]\right\} \mathrm{d} s \mathrm{~d} t \\
=-\int_{0}^{l}\left[m \delta R_{a}^{T} V_{a}+\delta \Phi_{B}^{T} J \Omega_{B}\right]_{t_{1}}^{t_{2}} \mathrm{~d} s
\end{gathered}
$$


where we have used the relation $\alpha^{T} \beta=-\frac{1}{2} \operatorname{tr}(\tilde{\alpha} \tilde{\beta})$. Substituting Eqs. (3), (10), and (11), into Eq. (12), and using the constant-strain discretization defined above, we obtain

$$
\begin{gathered}
\mathcal{M}_{s}(\bar{\gamma}, \bar{\kappa})\left\{\begin{array}{c}
\ddot{\bar{\gamma}} \\
\ddot{\bar{\kappa}}
\end{array}\right\}+\mathcal{C}_{s}(\bar{\gamma}, \bar{\kappa})\left\{\begin{array}{l}
\dot{\bar{\gamma}} \\
\dot{\bar{\kappa}}
\end{array}\right\}+\mathcal{K}_{s}\left\{\begin{array}{l}
\bar{\gamma} \\
\bar{\kappa}
\end{array}\right\}+\mathcal{M}_{\mathrm{gyr}}(\bar{\gamma}, \bar{\kappa})\left\{\begin{array}{l}
\dot{v}_{a} \\
\dot{\omega}_{a}
\end{array}\right\} \\
+\mathcal{C}_{\mathrm{gyr}}\left(\bar{\gamma}, \bar{\kappa}, \dot{\bar{\gamma}}, \dot{\bar{\kappa}}, \omega_{a}\right)\left\{\begin{array}{c}
v_{a} \\
\omega_{a}
\end{array}\right\}=\left\{\begin{array}{l}
f \\
m
\end{array}\right\}
\end{gathered}
$$

These are the dynamic equations in beam strains for given reference frame velocities and applied forces. The local displacements and rotations are obtained as a postprocessing step from Eqs. (8). The major advantage of this solution process is the constant stiffness matrix in the finite element discretization. Nonlinear static solutions will be then straightforward (the nonlinearity being in the straindisplacement relations) but also low-frequency dynamic situations, dominated by the stiffness terms.

\section{Intrinsic Beam Element}

A hybrid (or mixed) solution procedure can be also defined. Hodges [20] introduced a three-field solution, in which Eqs. (1-3) are all simultaneously enforced using Lagrange multipliers. This simplifies the solution process, as compared with the displacementbased solution of Eq. (5), by requiring simpler interpolation functions, but substantially increases the number of states in the problem. Moreover, retaining the rotation degrees of freedom as independent degrees is in general a costly option. A more interesting approach is a two-field solution [24] based on strains (or internal forces) and velocities along the reference line. In this case, Eq. (5) is complemented by an additional equation that results from eliminating the position vector and the rotation matrix in Eqs. (3). This is nothing more than a compatibility equation that imposes that the successive derivatives of the displacement/rotation field in time and space can be defined in any order. It results in the following system of equations [24]

$$
\begin{aligned}
& \dot{P}+\tilde{\Omega} P=F^{\prime}+\tilde{K} F+f \\
& \dot{H}+\tilde{\Omega} H+\tilde{V} P=M^{\prime}+\tilde{K} M+\left(\tilde{e}_{1}+\tilde{\gamma}\right) F+m \\
& \dot{\gamma}=V^{\prime}+\tilde{K} V+\left(\tilde{e}_{1}+\tilde{\gamma}\right) \Omega, \quad \dot{\kappa}=\Omega^{\prime}+\tilde{K} \Omega
\end{aligned}
$$

In Eq. (14) all vector components are projected into the local deformed frame $(B)$ and therefore the subindex has been dropped. Equations (14), together with the constitutive relations (2), provide a closed-form solution to the problem that only involves first-order differential equations. Displacements and rotations are dependent variables which can be obtained at each converged iteration by Eq. (8). The major advantage of this approach with respect to the previous one is that it reduces both the number of operations per iteration and the bandwidth of the matrices in the solution. Even though it doubles the number of unknowns, the simplicity of the resulting procedure have been found to provide significant reduction in computational cost for dynamics of open chains (aircraft-type geometries). This will be exemplified in the numerical examples in this paper. Note also that the free-body velocities, $v_{a}$ and $\omega_{a}$, and their derivatives no longer explicitly appear in the equilibrium equation. An alternative solution procedure, based on the finite element method, is considered instead of the finite differences solution in Hodges [24], and will be described in what follows in some detail. Equation (14), after substitution of Eq. (2), can be written in weak form as

$$
\begin{aligned}
& \int_{t_{1}}^{t_{2}} \int_{0}^{l}\left\{\delta R^{T}\left[m \dot{V}-m \tilde{\xi}_{\mathrm{cg}} \dot{\Omega}+m \tilde{\Omega}\left(V-\tilde{\xi}_{\mathrm{cg}} \Omega\right)-F^{\prime}-\tilde{K} F-f\right]\right. \\
& \quad+\delta \Phi^{T}\left[m \tilde{\xi}_{\mathrm{cg}} \dot{V}+J \dot{\Omega}+m \tilde{\xi}_{\mathrm{cg}} \tilde{\Omega} V+\tilde{\Omega} J \Omega-M^{\prime}-\tilde{K} M\right. \\
& \left.\quad-\left(\tilde{e}_{1}+\tilde{\gamma}\right) F-m\right]+\delta I^{T}\left[c_{\gamma f} \dot{F}+c_{\gamma m} \dot{M}-V^{\prime}-\tilde{K} V\right. \\
& \left.\left.\quad-\left(\tilde{e}_{1}+\tilde{\gamma}\right) \Omega\right]+\delta J^{T}\left[c_{\kappa f} \dot{F}+c_{\kappa m} \dot{M}-\Omega^{\prime}-\tilde{K} \Omega\right]\right\} \mathrm{d} s \mathrm{~d} t=0(15)
\end{aligned}
$$

where we have introduced the infinitesimal rotation $\widetilde{\delta \Phi}=C^{B a} \delta C^{a B}$, as well as the force and moment impulse variations, $\delta I$ and $\delta J$, respectively. They will also have to satisfy the boundary conditions. For instance, for a beam clamped at $s=0$ and free at $s=l$, it will be $\delta R(0)=0, \delta \Phi(0)=0, V(0)=0, \Omega(0)=0$, and $\delta I(l)=0, \delta J(l)=$ $0, F(l)=0, M(l)=0$, respectively. A finite element approximation of Eq. (15) is introduced now, as

$$
\begin{aligned}
& \left\{\begin{array}{l}
V \\
\Omega
\end{array}\right\}=N\left\{\begin{array}{l}
\bar{V} \\
\bar{\Omega}
\end{array}\right\}, \quad\left\{\begin{array}{l}
F \\
M
\end{array}\right\}=N\left\{\begin{array}{l}
\bar{F} \\
\bar{M}
\end{array}\right\} \\
& \left\{\begin{array}{l}
\delta R \\
\delta \Phi
\end{array}\right\}=W\left\{\begin{array}{l}
\delta \bar{R} \\
\delta \bar{\Phi}
\end{array}\right\}, \quad\left\{\begin{array}{l}
\delta I \\
\delta J
\end{array}\right\}=W\left\{\begin{array}{l}
\delta \bar{I} \\
\delta \bar{J}
\end{array}\right\}
\end{aligned}
$$

In the assembly process, a master-slave approach has been used here for elements which share a node: One of the elements was taken as reference and defines the degrees of freedom for a particular node in the global arrays, while the other elements become slaves on that shared node with its vector magnitudes defined by a constant rotation from the master element. The relative rotations between element variables at each node have been already embedded in Eq. (16). With these definitions, Eq. (15) can be rewritten as

$$
\begin{aligned}
& \mathcal{M} \frac{\mathrm{d}}{\mathrm{d} t}\left\{\begin{array}{l}
\bar{V} \\
\bar{\Omega}
\end{array}\right\}+\mathcal{Q}_{\mathrm{gyr}}(\bar{V}, \bar{\Omega})+\mathcal{Q}_{\text {stif }}(\bar{F}, \bar{M})=\mathcal{Q}_{\mathrm{ext}} \\
& \mathcal{T}_{1} \frac{\mathrm{d}}{\mathrm{d} t}\left\{\begin{array}{l}
\bar{F} \\
\bar{M}
\end{array}\right\}+\mathcal{T}_{\gamma}(\bar{F}, \bar{M})\left\{\begin{array}{l}
\bar{V} \\
\bar{\Omega}
\end{array}\right\}=0
\end{aligned}
$$

These are the equation of motion and the kinematic compatibility equation, respectively. The $\mathcal{T}$ matrices are

$$
\begin{aligned}
& \mathcal{T}_{1}=\int_{0}^{l} W^{T}\left[\begin{array}{cc}
\mathrm{c}_{\gamma f} & \mathrm{c}_{\gamma m} \\
\mathrm{c}_{\kappa f} & \mathrm{c}_{\kappa m}
\end{array}\right] N \mathrm{~d} s \\
& \mathcal{T}_{\gamma}=-\int_{0}^{l} W^{T} N^{\prime} \mathrm{d} s-\int_{0}^{l} W^{T}\left[\begin{array}{cc}
\tilde{K} & \tilde{e}_{1}+\tilde{\gamma} \\
0 & \tilde{K}
\end{array}\right] N \mathrm{~d} s
\end{aligned}
$$

The mass matrix in the equation of motion is a constant matrix, defined as

$$
\mathcal{M}=\int_{0}^{l} W^{T}\left[\begin{array}{cc}
m & -m \tilde{\xi}_{\mathrm{cg}} \\
m \tilde{\xi}_{\mathrm{cg}} & J
\end{array}\right] N \mathrm{~d} s
$$

The discrete gyroscopic, stiffness, and applied forces in the equation of motion are, respectively,

$$
\begin{aligned}
& \mathcal{Q}_{\mathrm{gyr}}=\int_{0}^{l} W^{T}\left\{\begin{array}{c}
m \tilde{\Omega}\left(V-\tilde{\xi}_{\mathrm{cg}} \Omega\right) \\
m \tilde{\xi}_{\mathrm{cg}} \tilde{\Omega} V+\tilde{\Omega} J \Omega
\end{array}\right\} \mathrm{d} s \\
& \mathcal{Q}_{\text {stif }}=\int_{0}^{l} W^{T}\left\{\begin{array}{c}
\tilde{F} K-F^{\prime} \\
\tilde{M} K-M^{\prime}+\tilde{F}\left(e_{1}+\gamma\right)
\end{array}\right\} \mathrm{d} s \\
& \mathcal{Q}_{\mathrm{ext}}=\int_{0}^{l} W^{T}\left\{\begin{array}{l}
f \\
m
\end{array}\right\} \mathrm{d} s
\end{aligned}
$$

where $F$ and $M$ are the internal forces and moments defined in Eq. (2). Equations (17) are complemented by a problem-dependent set of initial and boundary conditions. The system matrices $\mathcal{M}$ and $\mathcal{T}_{1}$ are constant (banded) matrices and only need to be inverted once and the problem can be easily posed as

$$
\dot{\mathbf{x}}=\mathbf{f}(\mathbf{x})+\mathbf{f}_{\mathrm{ext}}, \quad \mathbf{y}=\mathbf{g}(\mathbf{x})
$$

where the state and output variables are, respectively, $\mathbf{x}^{T}=$ $\left[\begin{array}{llll}\bar{V}^{T} & \bar{\Omega}^{T} & \bar{F}^{T} & \bar{M}^{T}\end{array}\right]$ and $\mathbf{y}^{T}=\left[\begin{array}{ll}\bar{R}^{T} & \bar{\Psi}^{T}\end{array}\right]$. In particular, $\mathbf{g}(\mathbf{x})$ is obtained from Eq. (8) if the internal forces and moments in each element are approximated by their value at the element midpoint. Note also that $\mathbf{f}(\mathbf{x})$ includes only quadratic nonlinearities, as the 
finite rotations only appear in the output equation, and this will improve the convergence rate of the solution algorithms as compared with the displacement- or strain-based elements introduced above. These equations define the intrinsic element ( $i$-beam element) and are therefore particularly suitable for integration together with the 1 st order different equations that describe the rigid-body dynamics of the flexible aircraft in flight mechanics analysis.

As in the case of the strain-based element, the intrinsic model that has been presented is only valid for open kinematic chains on a single support. Additional constraints (through, for example, Lagrange multipliers) would be needed for closed chains. The linearized expressions of Eqs. (17) can be easily obtained as

$$
\begin{gathered}
\mathcal{M} \frac{\mathrm{d}}{\mathrm{d} t}\left\{\begin{array}{c}
\Delta \bar{V} \\
\Delta \bar{\Omega}
\end{array}\right\}+C\left(V_{0}, \Omega_{0}\right)\left\{\begin{array}{l}
\Delta \bar{V} \\
\Delta \bar{\Omega}
\end{array}\right\} \\
+\mathcal{D}\left(F_{0}, M_{0}\right)\left\{\begin{array}{l}
\Delta \bar{F} \\
\Delta \bar{M}
\end{array}\right\}=\Delta \mathcal{Q}_{\mathrm{ext}} \\
\mathcal{T}_{1} \frac{\mathrm{d}}{\mathrm{d} t}\left\{\begin{array}{l}
\Delta \bar{F} \\
\Delta \bar{M}
\end{array}\right\}+\mathcal{T}_{\gamma}\left(F_{0}, M_{0}\right)\left\{\begin{array}{l}
\Delta \bar{V} \\
\Delta \bar{\Omega}
\end{array}\right\} \\
+\mathcal{T}_{v}\left(V_{0}, \Omega_{0}\right)\left\{\begin{array}{l}
\Delta \bar{F} \\
\Delta \bar{M}
\end{array}\right\}=0
\end{gathered}
$$

where the new matrices are

$$
\begin{aligned}
\mathcal{C} & =\int_{0}^{l} W^{T}\left[\begin{array}{cc}
m \tilde{\Omega} & m\left(\tilde{\xi}_{\mathrm{cg}} \tilde{\Omega}-2 \tilde{\Omega} \tilde{\xi}_{\mathrm{cg}}-\tilde{V}\right) \\
m \tilde{\xi}_{\mathrm{cg}} \tilde{\Omega} & \tilde{\Omega} J-\widetilde{J \Omega}-m \tilde{\xi}_{\mathrm{cg}} \tilde{V}
\end{array}\right] N \mathrm{~d} s \\
\mathcal{D} & =\int_{0}^{l} W^{T}\left[\begin{array}{cc}
0 & \tilde{F} \\
\tilde{F} & \tilde{M}
\end{array}\right]\left[\begin{array}{cc}
c_{\gamma f} & c_{\gamma m} \\
c_{\kappa f} & c_{\kappa m}
\end{array}\right] N \mathrm{~d} s \\
& -\int_{0}^{l} W^{T}\left[\begin{array}{cc}
\tilde{K} & 0 \\
\tilde{e}_{1}+\tilde{\gamma} & \tilde{K}
\end{array}\right] N \mathrm{~d} s-\int_{0}^{l} W^{T} N^{\prime} \mathrm{d} s \\
\mathcal{T}_{v} & =\int_{0}^{l} W^{T}\left[\begin{array}{cc}
\tilde{\Omega} & \tilde{V} \\
0 & \tilde{\Omega}
\end{array}\right]\left[\begin{array}{ll}
c_{\gamma f} & c_{\gamma m} \\
c_{\kappa f} & c_{\kappa m}
\end{array}\right] N \mathrm{~d} s
\end{aligned}
$$

Note that the linearized system matrices in Eq. (22) are not symmetric. For the particular case of nongyroscopic reference conditions (i.e., $V_{0}=0, \Omega_{0}=0$ ), the linear vibration characteristics are given by

$$
\begin{gathered}
{\left[\mathcal{D} \mathcal{T}_{1}^{-1} \mathcal{T}_{\gamma}+\omega^{2} \mathcal{M}\right]\left\{\begin{array}{c}
\phi_{V} \\
\phi_{\Omega}
\end{array}\right\}=0 \text { and }} \\
\left\{\begin{array}{c}
\phi_{F} \\
\phi_{M}
\end{array}\right\}=\frac{i}{\omega} \mathcal{T}_{1}^{-1} \mathcal{T}_{\gamma}\left\{\begin{array}{l}
\phi_{V} \\
\phi_{\Omega}
\end{array}\right\}
\end{gathered}
$$

Using the notation of Eq. (21), the linearized equations can finally be written in state-space form as

$$
\Delta \dot{\mathbf{x}}=\mathbf{A}\left(\mathbf{x}_{0}\right) \Delta \mathbf{x}+\Delta \mathbf{f}_{\mathrm{ext}}
$$

\section{Aerodynamic Modeling}

The unsteady aerodynamics (together with the aircraft weight loads) will provide the distributed applied forces and moments in either Eqs. (5), (13), and (21), or, as well as the total forces in the aircraft free-body Eqs. (4). In the present work, viscous drag forces are computed for each individual airfoil and assumed to be stationary, while potential-flow unsteady aerodynamics is assumed to compute the distributed lift, moment and induced drag of the different lifting surfaces. Two different unsteady aerodynamic models will be investigated here: As we are dealing with high-aspect ratio wings, a thin-strip solution will set the lower-order baseline model. However, large wing deflections may limit the applicability of the airfoil-based models and we will also consider a more involved UVLM to account for three-dimensional effects in the large wing deflections. They will be briefly described here for completion of the work.

\section{A. Thin-Strip Modeling}

In this case, wing airfoils are considered separately, with the unsteady forces and moments are corrected for tip effects from steady theory. We assume thin-airfoil theory, with an airfoil of chord $2 b$ and airfoil and wake in the $x-z$ plane and along $z=0$. For timedomain analysis, two different state-space approximations can be considered.

\section{Finite State Model Based on Glauert Expansions [16]}

The vertical induced velocity, $w_{\text {ind }}$, along the thin airfoil is given by

$$
w_{\text {ind }}=-\frac{1}{2 \pi} \int_{-b}^{b} \gamma_{b} \frac{1}{\left(x-x_{0}\right)} \mathrm{d} x_{0}-\frac{1}{2 \pi} \int_{b}^{\infty} \gamma_{w} \frac{1}{\left(x-x_{0}\right)} \mathrm{d} x_{0}
$$

where $\gamma_{b}$ and $\gamma_{w}$ are the (unknown) vortex distributions over the wing and wake, respectively. By applying the nonpenetration boundary condition on the airfoil, the vortex distribution over the wing section can be found. Peters et al. [16] obtained a solution method to the integral Eq. (26) based on Glauert's method, i.e., an expansion in Chebyshev polynomials. The induced velocity is decomposed into the velocity due to bound vorticity and the velocity due to the wake (or induced flow, $\lambda$, defined by the second integral above). Conservation of total vorticity leads to the Kutta condition to define the vorticity on the wake as a function of the changes of total bound vorticity on the airfoil, $\Gamma=\int_{-b}^{b} \gamma_{b} \mathrm{~d} x$. This defines the following condition on the induced flow [16]:

$$
\frac{\partial \lambda}{\partial t}+V \frac{\partial \lambda}{\partial x}=\frac{1}{2 \pi} \frac{\dot{\Gamma}}{b-x}
$$

After truncation, the components of the Glauert expansion of the inflow velocities define a natural set of aerodynamic states and are obtained after substitution into Eq. (26). Typically, four to eight states are necessary [16].

\section{Indicial Response Method [18]}

An alternate method [18] is to obtain the lift is from convolution of the response to a step change of angle of attack (Wagner's problem). Duhamel's integral for the resulting problem can be written as

$$
C_{L}^{c}(s)=2 \pi \alpha_{\mathrm{qs}}(0) \varphi(s)+2 \pi \int_{0}^{s} \frac{\mathrm{d} \alpha_{\mathrm{qs}}(\sigma)}{\mathrm{d} s} \varphi(s-\sigma) \mathrm{d} \sigma
$$

where $C_{L}^{c}$ is the circulatory lift coefficient, $\alpha_{\mathrm{qs}}$ is the quasi-steady effective angle of attack and $s$ is the reduced time. The usual Padé approximant to Wagner's function, $\varphi$, is taken: $\varphi(s)=1-A_{1} e^{-b_{1} s}-$ $A_{2} e^{-b_{2} s}$, where $A_{1}, A_{2}, b_{1}$ and $b_{2}$ are constants. This yields the following state-space description for the unsteady lift:

$$
\begin{aligned}
& \left\{\begin{array}{l}
\dot{x}_{1} \\
\dot{x}_{2}
\end{array}\right\}=\left[\begin{array}{cc}
-b_{1} & 0 \\
0 & -b_{2}
\end{array}\right]\left\{\begin{array}{l}
x_{1} \\
x_{2}
\end{array}\right\}+\left\{\begin{array}{l}
1 \\
1
\end{array}\right\} \alpha_{\mathrm{qs}}(s) \\
& C_{L}^{c}(s)=2 \pi\left[\begin{array}{ll}
A_{1} b_{1} & A_{2} b_{2}
\end{array}\right]\left\{\begin{array}{l}
x_{1} \\
x_{2}
\end{array}\right\}+\pi \alpha_{\mathrm{qs}}(s)
\end{aligned}
$$

As a result, the indicial response method adds only two aerodynamic states for each airfoil. Note that in discretized model of the aircraft, each airfoil will correspond to a beam finite element along the wing.

\section{B. Vortex-Lattice Modeling}

A VLM will define the unsteady aerodynamics model for either complex geometries or complex dynamics (resulting in nonplanar wake). The basics of the explicit unsteady VLM algorithm are described in [15]. As in other panel methods, such as the doublet lattice [25], elementary (singularity) solutions are distributed over a surface and the nonpenetration boundary condition is imposed at a number of control (collocation) points, leading to a system of 
algebraic equations. The VLM is also based on a thin-wing approximation, so both the elementary solutions and the collocation points are placed over the mean surface in lieu of the actual surface, thus effectively ignoring thickness effects.

Lifting surfaces and wakes (the latter obtained as part of the solution procedure) are discretized using vortex rings, i.e., quadrilateral elements composed by discrete vortex segments in a closed loop, along which the circulation strength, $\Gamma_{k}$, is constant. At the beginning of the motion, only the vortex rings covering the lifting surface exist (bound elements), and collocation points at which flowtangency will be imposed are associated to them. As the surface moves along its flight path, a force-free wake is formed, shed and convected. To accomplish this, each trailing-edge bound vortex ring sheds a wake panel, with circulation equal to the trailing-edge ring strength at the previous time step. This has been sketched in Fig. 2 . The wake elements are then freely convected and rolled up according to the local flow velocity, and thus generate a vortex ring lattice representing the shed wake. The circulation strength of the wake rings remains constant as they are moved away from the lifting surface, and they are allowed to roll up and stretch; albeit dissipation could also be accounted for by implementing any of various models for wake decay [26,27], this was not regarded as necessary for the applications under study.

The vorticity distribution of the bound vortex elements is determined by applying the nonpenetration boundary condition, requiring a zero velocity component normal to the solid surface at every collocation point $k$ :

$$
\sum_{l=1}^{K} a_{k l} \Gamma_{l}+\mathbf{v}_{k} \cdot \mathbf{n}_{k}=0
$$

for $l=1,2, \ldots, K$, where $K$ is the number of bound vortex rings of the lattice; $a_{k l}$ are the so-called aerodynamic influence coefficients, which stand for the normal velocity induced by vortex ring $l$ over collocation point $k$, computed by the Biot-Savart law (for unit circulation strength); $\mathbf{v}_{k}$ stands for the rest of the contributions to the velocity at collocation point $k$, encompassing kinematics of the wing (rigid-body motions plus deformations), wake vorticity and free stream; $\mathbf{n}_{k}$ is the unit normal vector of the panel. This equation is the three-dimensional equivalent to the induced-velocity equation for the airfoil, Eq. (26).

Application of the nonpenetration boundary condition, Eq. (30), at the $K$ collocation points leads to a system of algebraic equations, whereby the circulation strength of the bound vortex rings is determined at each time step. The aerodynamic loads can be obtained by computing the pressure across each panel, given by the numerical equivalent of the unsteady Bernoulli equation [15]:

$$
\Delta p_{k}=\rho\left\{\mathbf{v}_{k} \cdot \boldsymbol{\tau}_{i k} \frac{\Delta_{i} \Gamma_{k}}{\Delta c_{k}}+\mathbf{v}_{k} \cdot \boldsymbol{\tau}_{j k} \frac{\Delta_{j} \Gamma_{k}}{\Delta b_{k}}+\frac{\partial \Gamma_{k}}{\partial t}\right\}
$$

where $\boldsymbol{\tau}_{i k}$ and $\boldsymbol{\tau}_{j k}$ are the panel chordwise and spanwise tangential vectors, respectively; $\Delta c_{k}$ and $\Delta b_{k}$ are the panel chord and span, and subscripts $\Delta_{i} \Gamma_{k}$ and $\Delta_{j} \Gamma_{k}$ refer to the increment of circulation between two continuous vortex rings in the chordwise and spanwise directions, respectively.

\section{Numerical Studies}

Combinations of the previous structural and aerodynamics models provides a full description of the dynamics of flexible aircraft. In this work, our main concern is in the evaluation of the relative performance of all those different models in situations with large wing deflections. For that purpose, the different models have been implemented and verified and a number of tests have been carried out on them. We present here the most relevant results.

\section{A. Studies on the Structural Dynamic Models}

Previous work $[12,19,23]$ has extensively explored the details in the implementation of a strain-based model and this paper will focus on the other two. In particular, results obtained with two-noded displacement-based and intrinsic elements are presented. An important observation from the numerical tests is that, in a NewtonRaphson-based solution procedure, the intrinsic solution (based on first derivatives of the displacement/rotation field) shows quadratic convergence rates, as compared with linear one in the displacementbased model.

\section{Vertical Cantilever Beam on a Horizontal Moving Base}

The experimental results on very flexible Titanium beams obtained by Pai [28] will be used to verify the implementation of the time-domain solutions. The dimensions of the beam are $479.0 \times$ $50.8 \times 0.45 \mathrm{~mm}$, with mass density $4430 \mathrm{~kg} / \mathrm{m}^{3}$, Young's modulus $127 \mathrm{GPa}$, and Poisson's ratio 0.36. Experiments in [28] were performed with the undeformed beam in the vertical direction and clamped at its lower end on a horizontal moving base with harmonic oscillations of frequency $f_{\text {base }}$. This beam is highly flexible: the clamped beam in a horizontal position and subject to its own weight has a static tip deflection calculated to be $27.1 \%$ of its length. The first computed four natural frequencies for the horizontal beam are all bending modes (in parenthesis if including the weight): 1.71 (1.77), 10.66 (10.58), 29.96 (29.43), and $59.07(58.40) \mathrm{Hz}$.

Numerical simulations are presented fore the vertical beam on the horizontal moving base. The effect of gravity has been included as it affects the beam in its deformed position. For the intrinsic beam

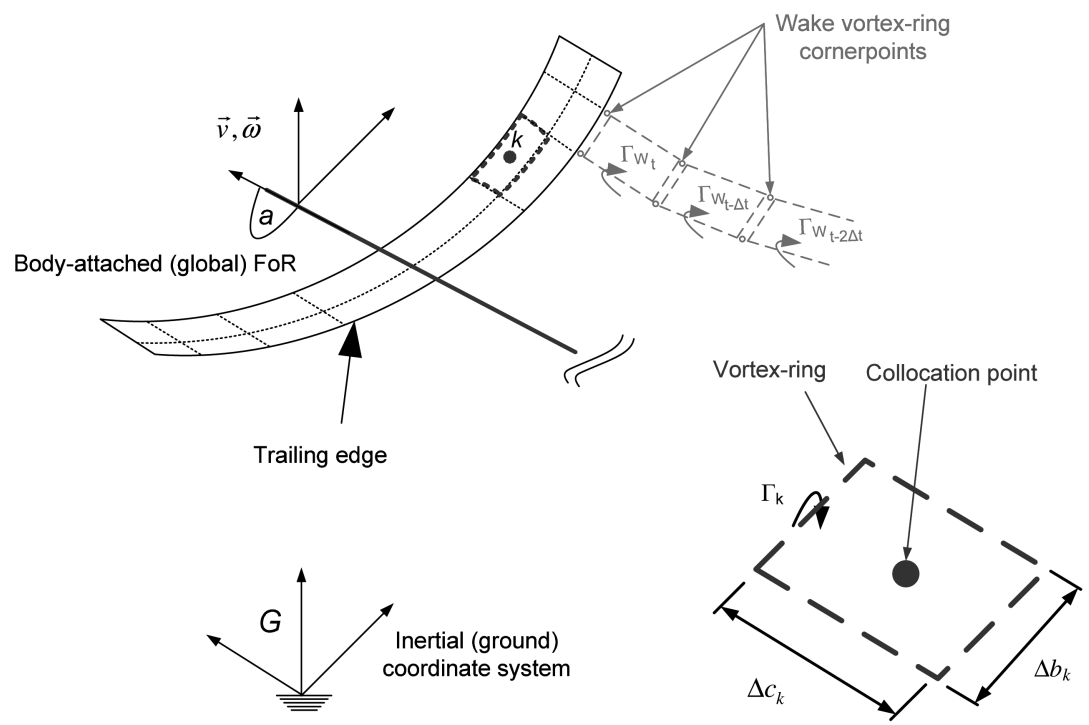

Fig. 2 Lifting surface and wake discretization using vortex ring elements. 
model ( $i$-beam element), they use a discretization in finite elements with linear interpolation and an implicit midpoint rule for time integration. This solution is equivalent to the finite difference solution process proposed by Hodges [24] and results in an energypreserving time integration scheme. A Newmark method was used for the second-order ODEs of the displacement-based model $(d$ beam). Figures $\underline{3}$ and 4 compare the linear and nonlinear intrinsic and displacement-based beam solutions with the envelope of the local velocities (absolute magnitude) measured by Pai [28] using a laser vibrometer. A discretization of 40 elements (about 1/10 of the wavelength of the fourth bending mode) was used in all cases and time step $\Delta t=0.01 / f_{\text {base }}$ (100 time steps per period of oscillation). Further refinement of the spatial or time discretizations did not give any significant improvement in accuracy. Results are normalized with the amplitude of the velocity at the base, $V_{\text {base }}$. They show very good agreement with the experimental data, both in the amplitudes of the beam motions and in the location of the nodes. The fourth natural frequency is about twice the value of the third one and the beam shows a two-to-one internal resonance when excited at $32 \mathrm{~Hz}$. As a result, even though beam displacements are small (with a maximum of about $2 \%$ of the beam span), there are differences between the linear and the nonlinear model due to the energy transfer between modes in the (nonlinear) internal resonance. This coincides with the experimental observations in [28]. Note finally that the linearization of the intrinsic model is carried out in the equations of motion, Eqs. (22), but not in the secondary evaluations of displacements and rotations, Eqs. (8), since those are only computed in postprocessing. As a result, the linearized intrinsic solution still keeps the nonlinearity between strain and displacements to better approximate the actual solution. This can be observed in Fig. 4, where velocity amplitudes in the linearized intrinsic model (on the left) are smaller than those of the displacement-based model (on the right). The internal resonance, however, can only be captured in the solution of the equations of motion and therefore the points of maximum amplitude are at the same (displaced) location in both linearized models in Fig. $\underline{4}$.

\section{45-Deg Bend Under Static Loads}

This is a curved cantilever beam in the shape of the arc of a circle. The bend spans $45 \mathrm{deg}$ of a circle in the $x-y$ plane and with a radius of $100 \mathrm{~m}$, and is subject to tip follower forces along the $z$ axis. This numerical test case was initially introduced in $[21,22]$ and will be used to compare the performance of the different beam models. The beam has a square cross section of side $1 \mathrm{~m}$, Young's modulus $E=10^{7} \mathrm{~Pa}$, and negligible Poisson ratio. Figure $\underline{5}$ shows the tip

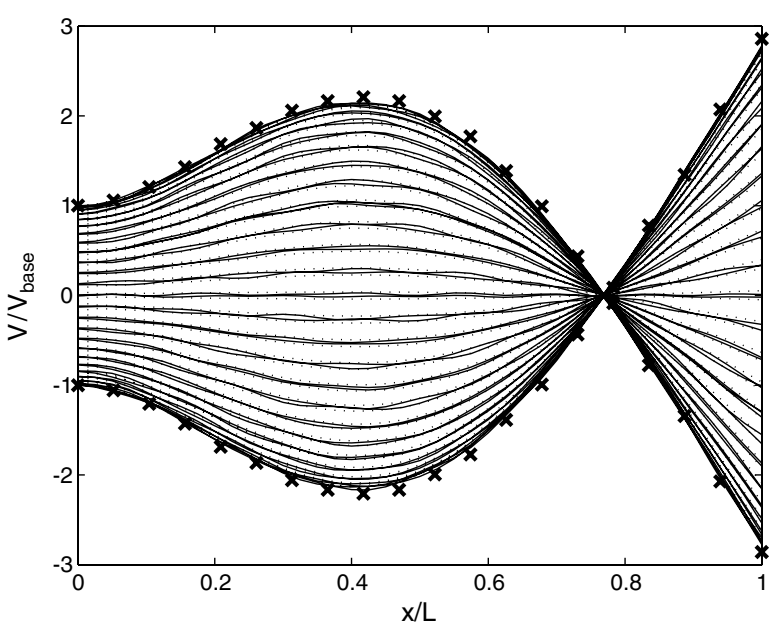

intrinsic model $\left[\mathrm{V}_{\text {base }}=0.1399 \mathrm{~m} / \mathrm{s}, \mathrm{f}_{\text {base }}=9 \mathrm{~Hz}\right]$

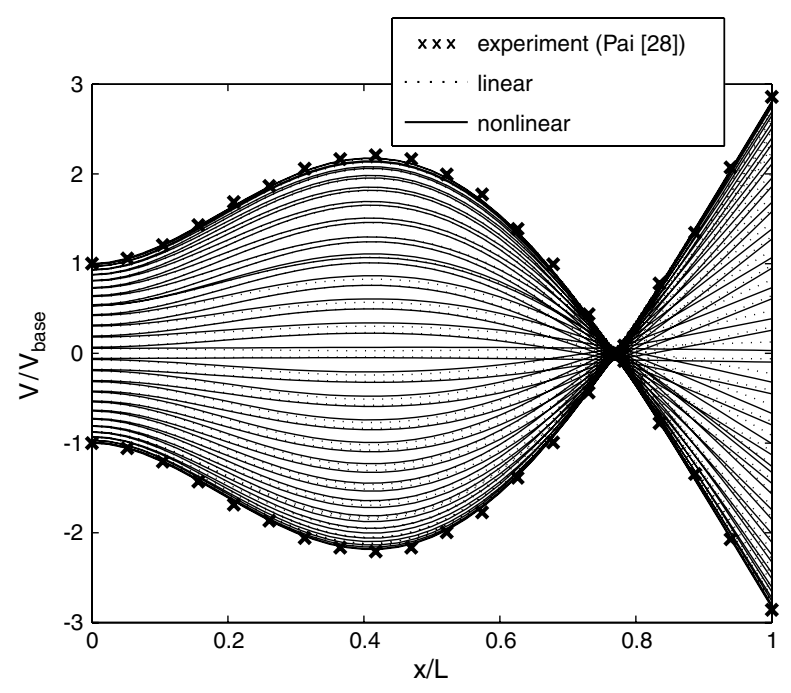

displacement-based [ $\mathrm{V}_{\text {base }}=0.1399 \mathrm{~m} / \mathrm{s}, \mathrm{f}_{\text {base }}=9 \mathrm{~Hz}$ ]

Fig. 3 Normal velocity profiles at 50 consecutive steps during one period of base oscillations (second bending).

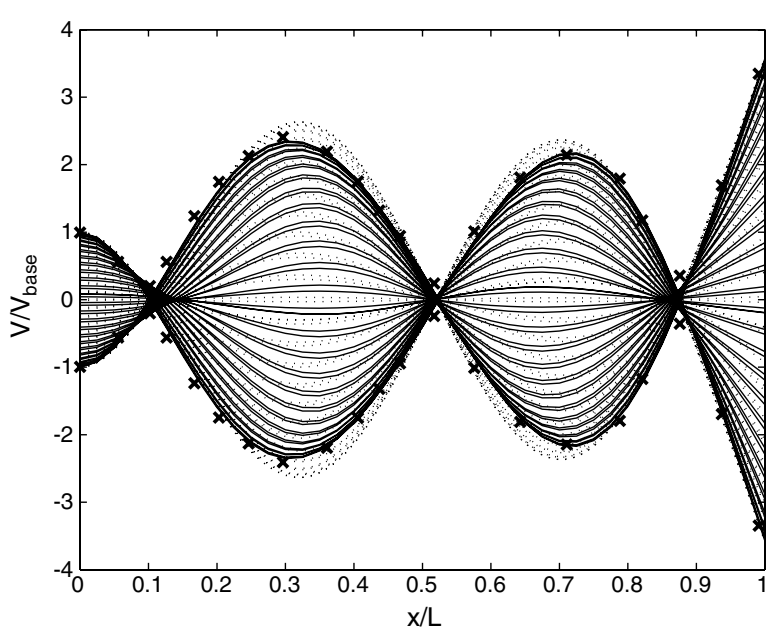

intrinsic model $\left[\mathrm{V}_{\text {base }}=0.3414 \mathrm{~m} / \mathrm{s}, \mathrm{f}_{\text {base }}=32 \mathrm{~Hz}\right]$

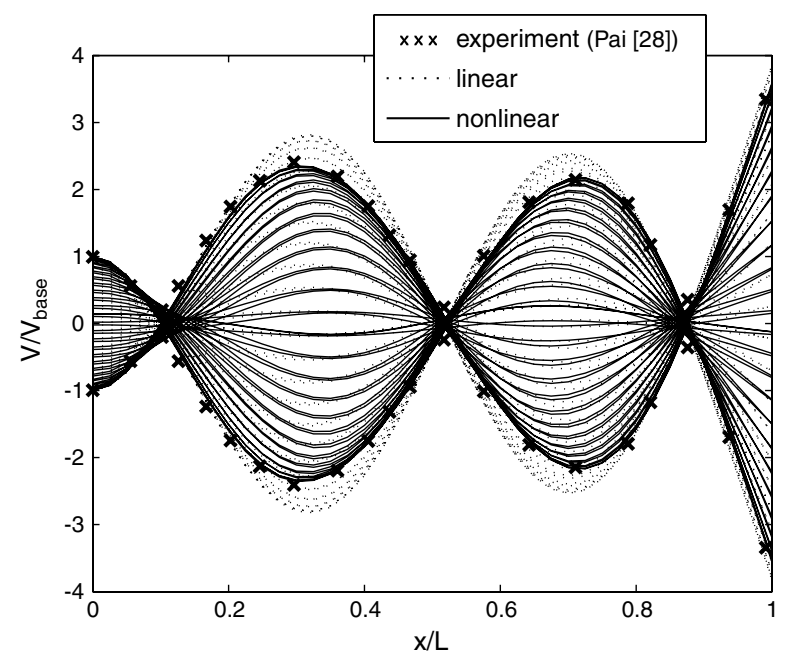

displacement-based $\left[\mathrm{V}_{\text {base }}=0.3414 \mathrm{~m} / \mathrm{s}, \mathrm{f}_{\text {base }}=32 \mathrm{~Hz}\right]$

Fig. 4 Normal velocity profiles at 50 consecutive steps during one period of base oscillations (fourth bending). 

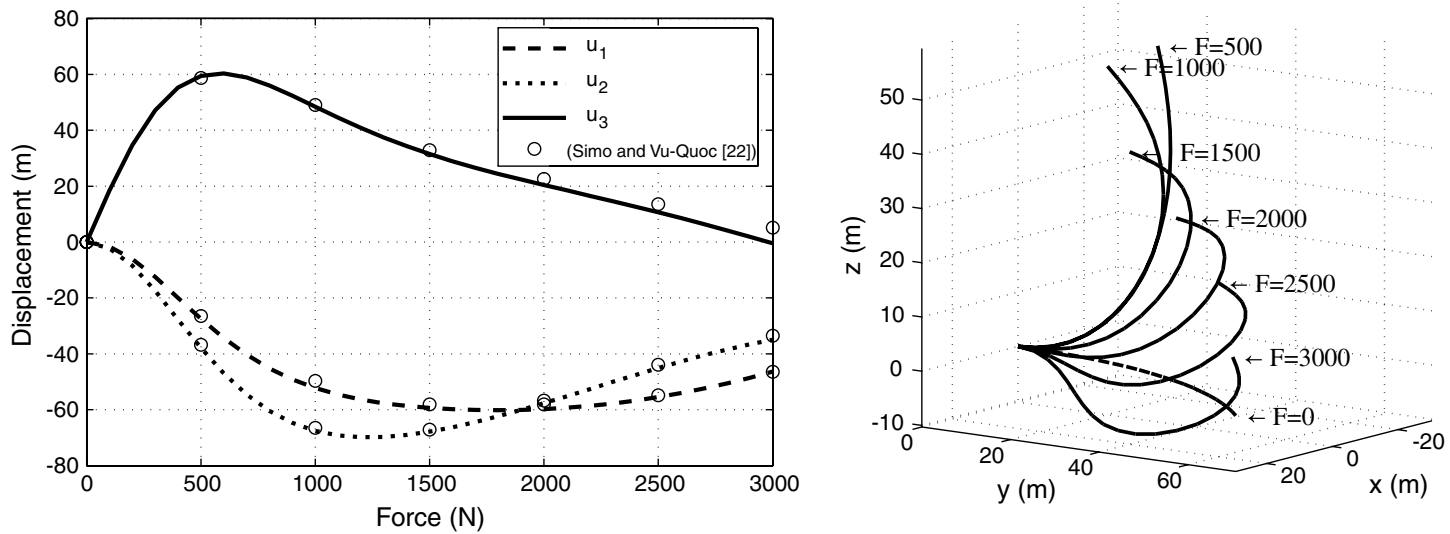

Fig. 5 Components of the tip displacement (left) and deformed shape (right) for different applied follower tip loads for the $45^{\circ}$ cantilever bend. Results obtained with the intrinsic beam model.

displacements and the deformed shape obtained with eight intrinsic elements (the figure on the right also shows, with $F=0$, the undeformed configuration). Results are compared with the displacement-based model of [22], which required eight load increments. The present intrinsic model obtains the solution without any need of applying the force in steps and with no more than 8 iterations for a result with a relative error smaller than $10^{-8}$. For dead loads, the intrinsic model needs some more iterations as it now needs to compute at each step the global rotation at the point of application of the load. As an example, the $45 \mathrm{deg}$ bend described above was subject to a tip vertical load of $300 \mathrm{~N}$. Both the displacement-based and the intrinsic model were used with 20-element two-noded discretizations and both were in good agreement with results in $[21,22]$. The increase in the number of elements was necessary to obtain the comparable levels of accuracy in the intrinsic and displacement model, as the intrinsic model carries out a numerical integration of the curvature vector along the span to compute the tip rotations. The displacement-based model required 22 load increments to converge and no less than 10 iterations on each increment, while the intrinsic element converged in 36 iterations without any load substepping. We should also remark that in this first implementation of the models, we have not yet considered the linearization of the state-dependent forces and there is still room for even better performance of the intrinsic beam model in this case. An additional advantage of this model is that it is based on simpler algorithms that require around half the number of operations per iteration.

\section{Wing-Body-Tail Configuration}

The advantage of the intrinsic model to consider large deformations can be even more dramatic if we complement it with model substructuring techniques that split the problem in the regions of small and large displacements, typically, fuselage/tail and wings, respectively. Such techniques also allow for the direct use of modal information of the fuselage obtained from more complex finite element models, to which geometrically-nonlinear models of the wings can be attached. To investigate this, a simpler wing-body-tail beam model is proposed. Fuselage length is $12 \mathrm{~m}$ and wing span is $30 \mathrm{~m}$. It has a T-tail 3-m high with a 6-m span. Isotropic properties were defined throughout, and the relevant constants are: $\mathrm{EA}=$ $5 \cdot 10^{8} \mathrm{~N}, \mathrm{kGA}=10^{8} \mathrm{~N}$, for extension and shear throughout the aircraft, respectively, bending stiffness equal to $\mathrm{EI}=5 \cdot 10^{6} \mathrm{Nm}^{2}$ at the fuselage and vertical tail, $\mathrm{EI}=5 \cdot 10^{5} \mathrm{Nm}^{2}$ in the horizontal tail, and $\mathrm{EI}=10^{6} \mathrm{Nm}^{2}$ along the wings. Constant normal (initially vertical) follower-forces are applied on the wings and horizontal tails and the model is clamped at the nose.

On this configuration, two models are studied. The first one is a standard model using displacement-based elements ( $d$-beams). A second model is obtained by using intrinsic elements only on the wings ( $i$-beams). The deformed configurations for different load levels are shown in Fig. 6, and they show very good matching of the results obtained from both models. However, the model with intrinsic elements for the wing runs 1 order of magnitude faster than the model with only displacement-based elements. For a $3000 \mathrm{~N} / \mathrm{m}$ load level, the latter needs at least 23 load subiterations, while the former needs only nine (larger than before because $d$-beams are still present in the model). In terms of computer time, for that case the use of $d$-beams only took 4.96 times more than when wings were modeled using the $i$-beams. Further improvement could be obtained if the elements in the fuselage and tail are linearized, but this has not been investigated here.

\section{B. Studies on the Aerodynamic Models}

As discussed in the introduction, the use of aerodynamic models based on two-dimensional strip theory is the most common in the literature for flight dynamics modeling of flexible aircraft with highaspect ratio wings. However, it assumes small deformations and a flat wake, and this reduces the reliability of the results as deflections grow larger and depart from the linear regime. To better understand the applicability of the different models, we will, first, investigate the performance of the different state-space airfoil models to capture unsteady aerodynamic loads, and then we will compare them with the VLM for increasing wing bending amplitudes and frequencies.

\section{Comparison of Finite State Airfoil Aerodynamic Models}

To quantify the error of the finite state and indicial response methods, Theodorsen's exact solution in the frequency domain will define the baseline for comparison [29]. Plotting lift coefficient against angle of attack for these harmonic oscillations results in a hysteresis ellipse which arises from a phase difference between the angle of attack and the lift. Figure 7 includes the relative error in the amplitude $\left(C_{L, \max } / \alpha_{\max }\right)$ and phase for both two-dimensional

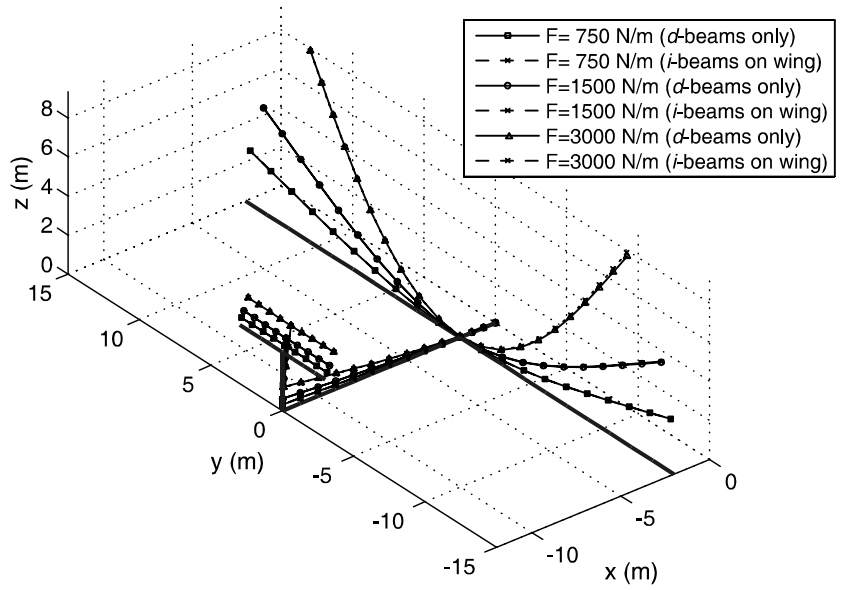

Fig. 6 Deformation of wing-body-tail configuration for follower distributed load on wing and tail. 

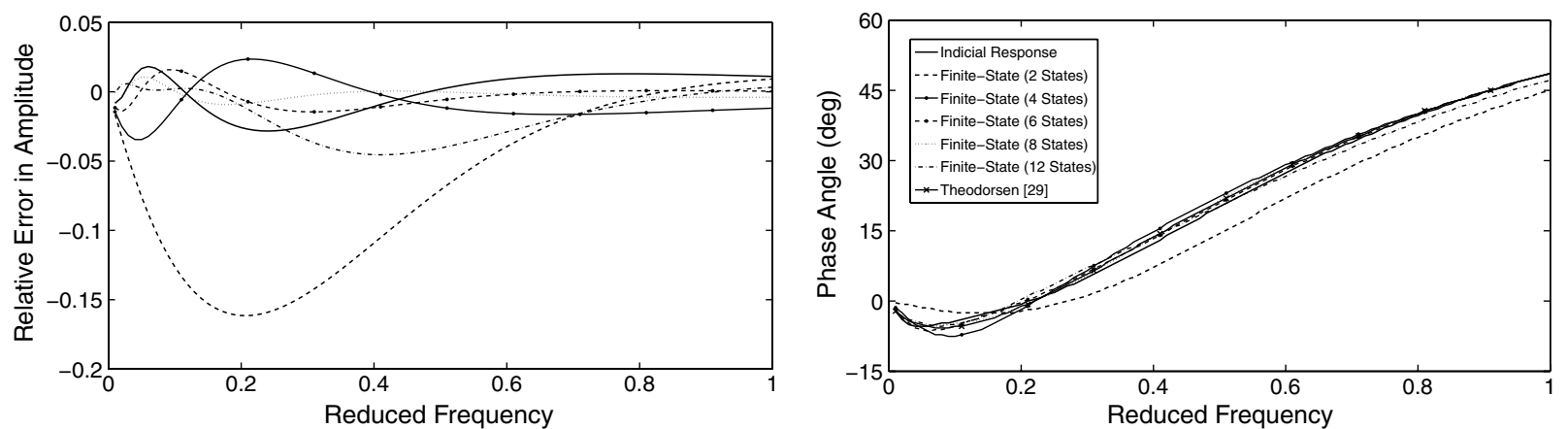

Fig. 7 Relative error in amplitude (left) with respect to Theodorsen's solution [29], and value of the phase (right) of the lift coefficient on airfoil with unit harmonic pitch oscillations at varying frequencies.

methods with Theodorsen's solution over a range of reduced frequencies, $k$.

It can be seen that the relative error is generally lower for very low and high frequencies, and greatest in both methods for the reduced frequency range $0.1-0.8$. The indicial response method has much lower relative error than the finite state method for equal number of states (i.e., two terms), and is comparable in magnitude with the error seen from four to six terms. It can also be seen that as the number of expansion terms exceeds eight, the results become less accurate for the finite state method indicating a failure to converge. The reason for this comes from an approximation needed for the zero-order term in the Glauert's expansion of the inflow $\left(\lambda_{0}\right)$ needed by the theory. This was reviewed in [17], including alternate methods to the current approximation (referred to as the augmented method [16]). While convergence of the augmented method was not discussed in [17], an alternate method based on a binomial approximation is proposed that requires around 50 states for a similar accuracy to eight states of the augmented one. Results here show that the two states in the indicial approach give an error no larger than $2 \%$ of the exact solution and therefore will often be a good first approximation to compute unsteady lift on airfoils.

\section{Aerodynamic Forces on a Flapping and Twisting Wing in Forward Flight}

A UVLM has been implemented by the authors and it will be verified first against results for the complex flapping kinematics defined in [30]. In that work, Stanford and Beran [30] explored the coupling between flapping and dynamic twisting in a sensitivity analysis of flapping flight using the UVLM. A large-amplitude pure rigid-body flapping (flapping angle $\beta=45^{\circ} \cos (\omega t)$ ) and a combination of flapping plus a first twisting mode (tip twist angle $\left.\theta=-45^{\circ} \sin (\omega t)\right)$ are investigated, on two rectangular symmetric wings with rigid angle of attack $\alpha=5^{\circ}$. Each wing has an aspect ratio $\mathrm{AR}=3$ and a NACA 8300 airfoil. Motions that are representative of the quasi-steady $(k=0.1)$ and unsteady $(k=0.75)$ regimes, respectively, are considered. Each flapping cycle of period $T=$
$2 \pi / \omega$ is divided into 40 time steps, and the wing is discretized using six panels chordwise and 10 along the semispan. Note that the comparison is made for code verification purposes only, and not to investigate flapping flight, since leading-edge separation is very likely to occur at the conditions of the analysis. Moreover, the expression used in [30] to compute the pressure jump across the panels, differs slightly from that published in [15], and given by Eq. (31), since the wake vorticity is not taken into account when computing the downwash, $\mathbf{v}_{k}$. To validate the code, results of [30] are compared with the present implementation of the UVLM (including wake vorticity in the computation of pressure), together with the modified code (no wake vorticity). Figure 8 shows the lift coefficient for quasi-steady (left) and unsteady (right) motions. In the quasisteady case $(k=0.1)$, the influence of including the wake on the computation of downwash, $\mathbf{v}_{k}$, is not significant, but the current implementation, despite capturing the qualitative behavior, presents quantitative discrepancies with respect to [30]. On the contrary, the influence of the wake is substantial in the unsteady case $(k=0.75)$, but the modified version (without wake) follows very closely [30]. Very good agreement was found, particularly considering that the there are a number of parameters, such as the definition of the local lift vector or the location of the shed wake, for which different values can be chosen in a UVLM implementation. In what follows, results will be presented including wake vorticity in Eq. (31), as done in [15].

\section{Three-Dimensional Effects in Wings with Large Time-Dependent Deflections}

The UVLM is compared against the two-dimensional unsteady airfoil models to evaluate the impact of large wing deflections on the aerodynamic loads. A parabolic bending mode has been prescribed along the wing (no twisting) and results are presented for different reduced frequencies, $k$, amplitudes of wing-tip deflection, $A_{\text {tip }}$, and wing aspect ratio, AR. Figures 9 and 10 show the maximum lift coefficient of half-wing for a whole flapping cycle and compare the UVLM, unsteady two-dimensional aerodynamics, and unsteady
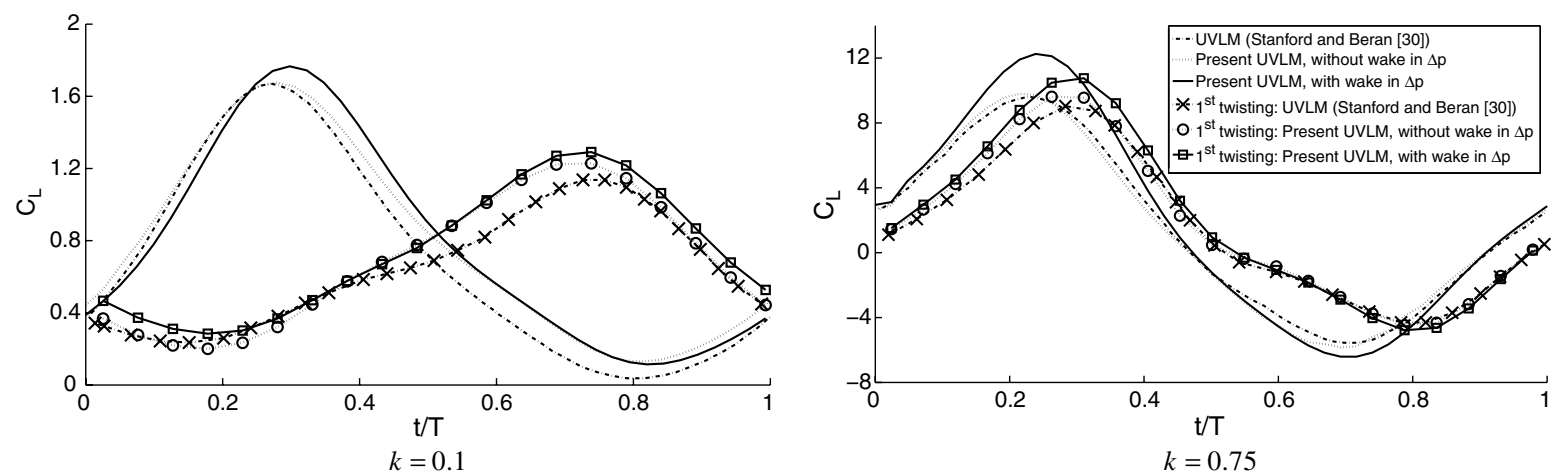

Fig. 8 Lift coefficient during a single rigid-body flapping cycle for a wing of aspect ratio three for varying reduced frequencies. UVLM results with and without wake vorticity in the computation of the pressure jump in Eq. $(\underline{31})$. NACA 8300 airfoil, aspect ratio three, $\alpha=5^{\circ}, \beta=45^{\circ} \cos (\omega t)$, $\theta=-45^{\circ} \sin (\omega t)$. 

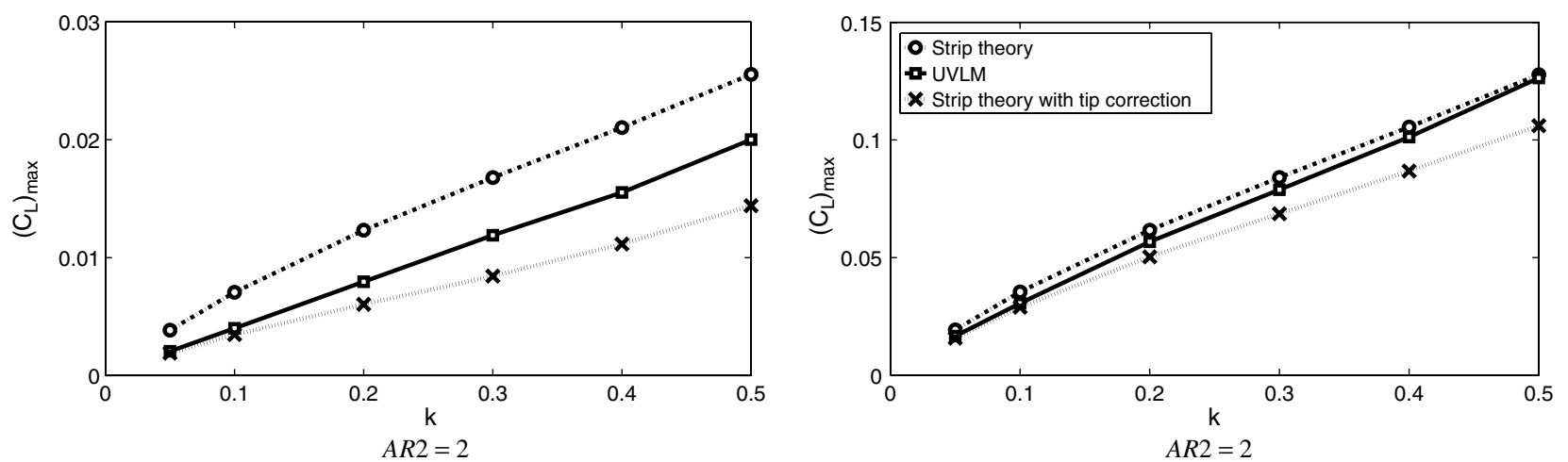

Fig. 9 Maximum lift coefficient for harmonic spanwise-parabolic deflections of a rectangular wing for varying oscillation frequency $\left(A_{\text {tip }} / d=0.01\right)$.

two-dimensional aerodynamics with the static lift slope at the tip airfoils corrected to match the steady vortex lattice. First, the influence of the reduced frequency is analyzed in Fig. 9 for a wing-tip deflection amplitude of $A_{\text {tip }}=0.01 \cdot d$, where $d$ is the semispan of the wing (root-to-tip distance). Two different wing semispan aspect ratios are considered: $\mathrm{AR}=2$ and $\mathrm{AR}=10$. For $\mathrm{AR}=2$, strip theory disagrees over the whole range, since for this relatively small aspect ratio three-dimensional effects are significant. If the tip correction is applied, results agree well with the VLM at very low reduced frequencies (quasi-steady case). However, as the frequency increases, so it does the discrepancy between corrected strip theory and UVLM. The steady corrections overestimate the tip effects in unsteady flows. For $\mathrm{AR}=10$, a similar behavior can be observed for very low reduced frequencies. However, at higher reduced frequencies the uncorrected strip theory is closer to the UVLM, which is due to the increase in aspect ratio, leading to a more twodimensional-like behavior. Therefore, for low-to-moderate reduced frequencies and small deformations, the disagreement with respect to the three-dimensional model can be considerable, both for corrected and uncorrected strip theory.

The effect of larger deformations is studied next. Figure 10 presents the maximum lift coefficient for a half-wing of aspect ratio $\mathrm{AR}=2$ and $\mathrm{AR}=10$. Wing-tip deflection amplitudes vary from 1 to

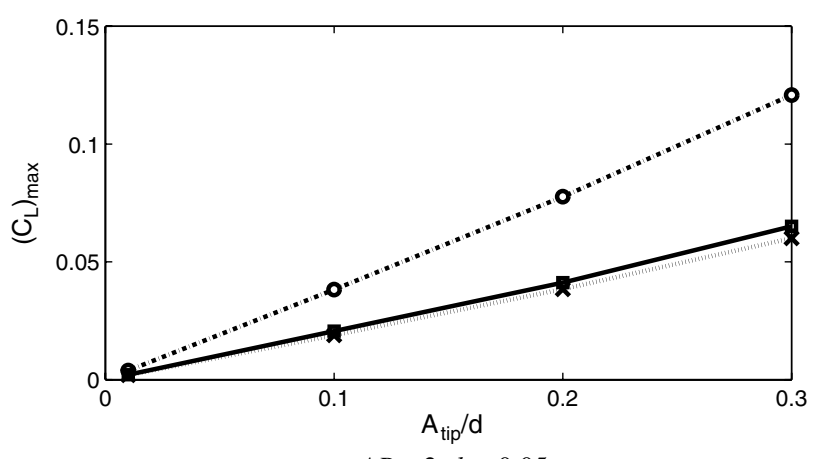

$A R=2, k=0.05$

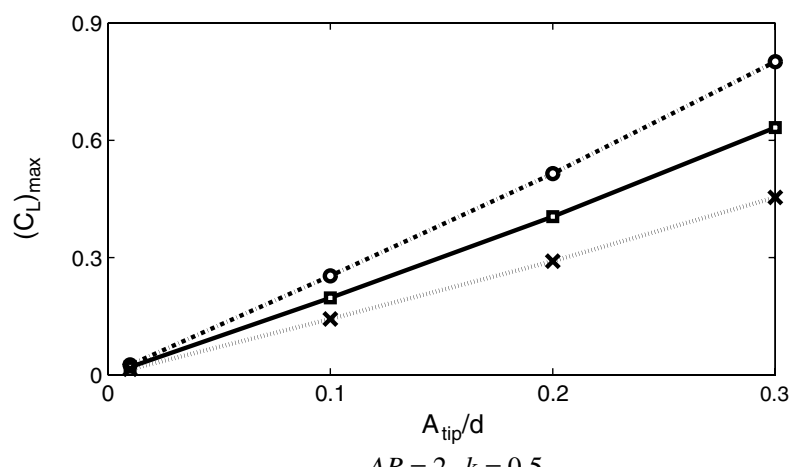

$30 \%$ of the semispan. Unsteady oscillations with reduced frequencies of $k=0.5$ and $k=0.2$, respectively, are compared with a quasisteady case $(k=0.05)$. The maximum frequency considered depends on the aspect ratio, since high oscillation frequencies, together with large spans and tip deflections would lead to unrealistic situations. Moreover, beyond certain values of the induced angle of attack (which is linearly proportional to the above three parameters), the results obtained by the UVLM are no longer reliable: as the flapping velocity increases, the wake-shedding from the wing-tip (in the spanwise direction) would become important, and since this is not being accounted for, the UVLM would overestimate the lift. The agreement of the corrected strip theory and the UVLM is reasonably good for the quasi-steady case for the deformations considered, for both aspect ratios. In contrast, the discrepancy is very important for the unsteady case over most of the range. For low aspect ratios (Fig. 10, left), while strip theory overpredicts the lift, the corrected approximation underestimates it by a significant margin, and only for small deformations is there any agreement. For the larger aspect ratio case (Fig. 10, right), even though the uncorrected model yields a better accuracy, none of the two-dimensional methods is capable of capturing the effects of time-dependent geometrically-nonlinear deformations. They are expected to be particularly important for the dynamics of VFA passing through strong gusts.
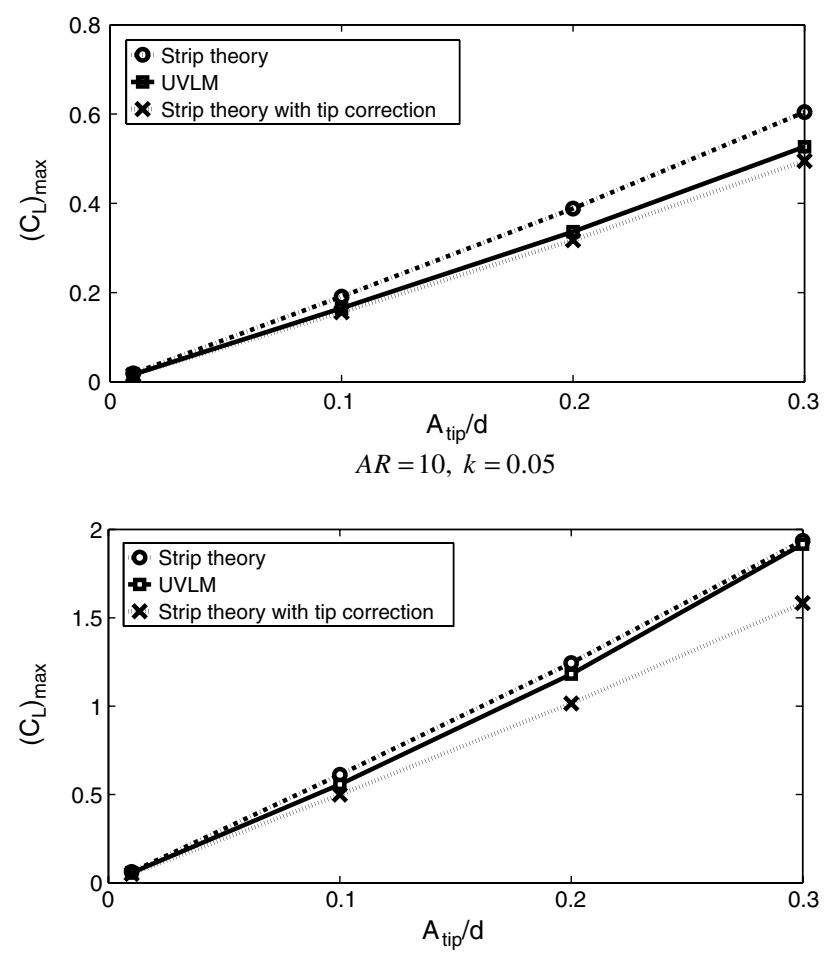

$A R=10, k=0.2$

Fig. 10 Maximum lift coefficient for harmonic spanwise-parabolic deflections of a rectangular wing for varying tip amplitude. 


\section{Conclusions}

This work has evaluated different structural and aerodynamics models as a step towards building numerically-efficient methodologies to investigate the dynamics of very flexible UAVs. The solution to the geometrically-nonlinear beam equations was obtained with three different sets of independent degrees of freedom. It has been found that the computational cost of conventional displacement-based models can be significantly reduced by using a description based on the intrinsic beam equations, in which velocities and internal forces define the independent structural states of the vehicle. A strain-based model, based on beam strains and their time derivatives offers an alternative efficient method. As with the intrinsic model, it does not need to integrate finite rotations, and in this case it also preserves the symmetry of the system (and the second-order differential-equation form). This work has expanded previous descriptions of the strain-based model to include the shear strains as independent degrees of freedom. It has also presented a finite element solution methodology for the intrinsic model. Results have shown the effectiveness of these nonconventional beam models to address geometrically-nonlinear problems with a small number of joints (aircraftlike geometries). This was exemplified with static problems with large displacements and dynamic problems with internal resonances. Linear structural results in those cases need to be refined and it was shown the efficiency of strain-based approaches to provide the nonlinear description. Furthermore, the first-order nonlinear state-space form of the discrete intrinsic beam equations provides a natural description of the vehicle structural dynamics for integration in flight mechanic analysis.

The studies on the aerodynamic modeling have looked into the numerical efficiency and the importance of three-dimensional effects on wings with large deflections. First, two state-space thin-airfoil models were compared, namely a model based on a Glauert expansion of the inflow velocity field and an indicial response based on the usual Padé approximant to Wagner's step response. It was found that the second one performs better at low reduced frequencies for a given number of states, which is in accordance to previous findings. The indicial method was then compared with an unsteady vortex lattice for wings undergoing large bending displacements. It was found that at low frequencies, the three-dimensional tip effects can have a relatively large influence but that this correction is no longer needed for high aspect ratio wings at larger frequencies. Although simpler two-dimensional unsteady airfoil models can provide an appropriate estimation of the aerodynamic loads in a wide range of the expected kinematics of large Unmanned Aerial Vehicles (UAVs), they may not suffice for large-amplitude and frequency wing dynamics. However, dynamic stall has not been considered here, but it can be critical on very flexible wings. In that was needed, semi-empirical models can be more easily introduced into the twodimensional models than on a vortex lattice discretization. Finally, the VLM can predict the shape of the wake, which would be an important asset in simulations of flexible UAVs where wake-tail interference is likely to occur.

The different models investigated in this work have been developed within a single analysis framework, as independent modules that can be used to describe the coupled flight dynamics and aeroelasticity of flexible aircraft. They offer a low-order description to introduce flexible effects in the prediction of performance and stability of those vehicles, and in the design of their flight-control system. The outcomes of the present study provide guidelines for selection of the appropriate structural and aerodynamics models in such studies.

\section{Acknowledgments}

The work of Joseba Murua is funded by a grant by the Basque Government. The work of Robbie Cook is supported by a U.K. Engineering and Physical Sciences Research Council Industrial Cooperative Award in Science and Engineering sponsored by QinetiQ, with Ian Roberts as the technical monitor. The first author is particularly grateful to Carlos Cesnik from the University of Michigan for a number of very helpful discussions to identify the different structural solution approaches addressed in this work. The assistance and guidance of Mike Graham from Imperial College in the implementation of the unsteady vortex lattice method has also been extremely valuable. Finally, Bret Stanford, from Air Force Research Laboratory, kindly gave us very useful recommendations to carry out the simulations of the flapping wing case.

\section{References}

[1] Rodden, W. P., and Love, J. P., "Equations of Motion of a Quasisteady Flight Vehicle Utilizing Restrained Static Aeroelastic Characteristics," Journal of Aircraft, Vol. 22, No. 9, 1985, pp. 802-809. doi: $10.2514 / 3.45205$

[2] Meirovitch, L., and Tuzcu, I., "Unified Theory for the Dynamics and Control of Maneuvering Flexible Aircraft," AIAA Journal, Vol. 42, No. 4, 2004, pp. 714-727 doi:10.2514/1.1489

[3] Baldelli, D. H., Chen, P. C., and Panza, J., "Unified Aeroelastic and Flight Dynamic Formulation via Rational Function Approximations,' Journal of Aircraft, Vol. 43, No. 3, 2006, pp. 763-772. doi:10.2514/1.16620

[4] Noll, T. E., Ishmael, S. D., Henwood, B., Perez-Davis, M. E., Tiffany, G. C., Madura, J., Gaier, M., Brown, J. M., and Wierzbanowski, T., "Technical Findings, Lessons Learned, and Recommendations Resulting from the Helios Prototype Vehicle Mishap," NATO/RTO AVT-145 Workshop on Design Concepts, Processes and Criteria for UAV Structural Integrity, Florence, Italy, 14-18 May 2007.

[5] Van Schoor, M. C., and von Flotow, A. H., "Aeroelastic Characteristics of a Highly Flexible Aircraft," Journal of Aircraft, Vol. 27, No. 10, 1990, pp. 901-908. doi: $10.2514 / 3.45955$

[6] Drela, M., "Integrated Simulation Model for Preliminary Aerodynamic, Structural, and Control-Law Design of Aircraft," AIAA Paper No. 1999-1394, 1999.

[7] Patil, M. J., Hodges, D. H., and Cesnik, C. E. S., "Nonlinear Aeroelastic Analysis of Complete Aircraft in Subsonic Flow," Journal of Aircraft, Vol. 37, No. 5, 2000, pp. 753-760. doi:10.2514/2.2685

[8] Shearer, C. M., and Cesnik C. E. S., "Nonlinear Flight Dynamics of Very Flexible Aircraft," Journal of Aircraft, Vol. 44, No. 5, 2007, pp. 1528-1545. doi: $10.2514 / 1.27606$

[9] Wang, Z., Chen, P. C., Liu, D. D., and Mook, D. T., "NonlinearAerodynamics/Nonlinear-Structure Interaction Methodology for a High-Altitude Long-Endurance Wing," Journal of Aircraft, Vol. 47, No. 2, 2010, pp. 556-566. doi: $10.2514 / 1.45694$

[10] Chang, C. S., Hodges, D. H., and Patil, M. J., "Flight Dynamics of Highly Flexible Aircraft," Journal of Aircraft, Vol. 45, No. 2, 2008, pp. 538-545. doi: $10.2514 / 1.30890$

[11] Palacios, R., Cesnik, C. E. S., and Reichenbach, E. Y., "Re-Examined Structural Design Procedures for Very Flexible Aircraft," Proceedings of the 13th International Forum of Aeroelasticity and Structural Dynamics, Stockholm, Sweden, 18-20 June 2007.

[12] Cesnik, C. E. S., and Brown, E. L., "Modeling of High Aspect Ratio Active Flexible Wings for Roll Control," AIAA Paper No. 2002-1719, 2002.

[13] Palacios, R., and Cesnik, C. E. S., "Cross-Sectional Analysis of NonHomogeneous Anisotropic Active Slender Structures," AIAA Journal, Vol. 43, No. 12, 2005, pp. 2624-2638. doi:10.2514/1.12451

[14] Jung, W., and Reimerdes, H. G., "Concepts for Reduced Structural Models of Airplane Wings in Aeroelasticity," Flow Modulation and Fluid-Structure Interaction at Airplane Wings, edited by J. Ballmann, Notes on Numerical Fluid Mechanics and Multidisciplinary Design, Vol. 84, Springer-Verlag, Berlin, 2003, pp. 249-273.

[15] Katz, J., and Plotkin, A., Low-Speed Aerodynamics, Cambridge Univ. Press, New York, 2001, pp. 369-447.

[16] Peters, D. A., Karunamoorthy, S., and Cao, W., "Finite State Induced Flow Models. Part 1: Two-Dimensional Thin Airfoil," Journal of Aircraft, Vol. 32, No. 2, 1995, pp. 313-322. doi: $10.2514 / 3.46718$

[17] Peters, D. A., "Two-Dimensional Incompressible Unsteady Airfoil Theory: An Overview," Journal of Fluids and Structures, Vol. 24, No. 3, 2008, pp. 295-312.

doi:10.1016/j.jfluidstructs.2007.09.001

[18] Leishman, J. G., "Unsteady Lift of a Flapped Airfoil by Indicial 
Concepts," Journal of Aircraft, Vol. 31, No. 2, 1994, pp. 288-297. doi: $10.2514 / 3.46486$

[19] Shearer, C. M., and Cesnik, C. E. S., "Modified Generalized- $\alpha$ Method for Integrating Governing Equations of Very Flexible Aircraft," AIAA Paper No. 2006-1747, 2006.

[20] Hodges, D. H., "A Mixed Variational Formulation Based on Exact Intrinsic Equations for Dynamics of Moving Beams," International Journal of Solids and Structures, Vol. 26, No. 11, 1990, pp. 1253-1273. doi:10.1016/0020-7683(90)90060-9

[21] Géradin, M., and Cardona, A., "Flexible Multibody Dynamics," A Finite Element Approach, Wiley, Chichester, England, U.K., 2001, pp. 133-134.

[22] Simo, J. C., and Vu-Quoc, L., "A Three-Dimensional Finite: Part 2: Computational Aspects," Computer Methods in Applied Mechanics and Engineering, Vol. 58, No. 1, 1986, pp. 79-116. doi:10.1016/0045-7825(86)90079-4

[23] Cesnik, C. E. S., and Su, W., "Nonlinear Aeroelastic Modeling and Analysis of Fully Flexible Aircraft," AIAA Paper No. 2005-2169, 2005

[24] Hodges, D. H., "Geometrically Exact, Intrinsic Theory for Dynamics of Curved and Twisted Anisotropic Beams," AIAA Journal, Vol. 41, No. 6 , 2003, pp. 1131-1137. doi: $10.2514 / 2.2054$
[25] Rodden, W. P., "The Development of the Doublet-Lattice Method," Eighth International Forum on Aeroelasticity and Structural Dynamics, Rome, Italy, 17-20 June 1997.

[26] Fritz, T. E., and Long, L. N., "Object-Oriented Unsteady Vortex Lattice Method for Flapping Flight," Journal of Aircraft, Vol. 41, No. 6, 2004, pp. $1275-1290$. doi: $10.2514 / 1.7357$

[27] Saban, D., and Whidborne, J. F., "Modeling of Wake Vortex Effects for Unmanned Air Vehicle Simulations," AIAA Paper No. 2009-5686, 2009.

[28] Pai, P. F., Highly Flexible Structures: Modeling, Computation, and Experimentation, AIAA Education Series, AIAA, Reston, VA, 2007, pp. 392-399.

[29] Theodorsen, T., "General Theory of Aerodynamic Instability and the Mechanism of Flutter," NACA Rept. No. 496, 1935.

[30] Stanford, B. K., and Beran, P. S., "Analytical Sensitivity Analysis of an Unsteady Vortex Lattice Method for Flapping Wing Optimization," Journal of Aircraft, Vol. 47, No. 2, 2010, pp. 647-662. doi: $10.2514 / 1.46259$
K. Willcox Associate Editor 